\title{
Limit Theorems for Weighted Sums of Infinite Variance Random Variables Attracted to Integrals of Linear Fractional Stable Motions
}

\author{
Makoto MAEJIMA and Sakurako SUZUKI
}

Keio University and Ochanomizu University

(Communicated by A. Tani)

\begin{abstract}
Let $\left\{\xi_{j}\right\}_{j \in \mathbf{Z}}$ be a sequence of random variables which belong to the domain of attraction of a linear fractional stable motion $\left\{\Delta_{H, \alpha}(t)\right\}$ with infinite variance. We study the convergence of weighted sums $I_{n}(f):=$ $A_{n} \sum_{j \in \mathbf{Z}} f(j / n) \xi_{j}$ with a suitable scaling $A_{n}$, to $I(f):=\int_{-\infty}^{\infty} f(u) d \Delta_{H, \alpha}(u)$ in distribution under suitable assumptions on a class of deterministic functions $f$. We also show that if $\left\{f_{t}, t \geq 0\right\}$ are the kernel functions from the "moving average" representation of a linear fractional stable motion with another index $H^{\prime}$, then $\left\{I_{n}\left(f_{t}\right)\right\}$ converges to a linear fractional stable motion $\left\{\Delta_{H+H^{\prime}-1 / \alpha, \alpha}(t)\right\}$.
\end{abstract}

\section{Introduction}

We call $\{X(t), t \geq 0\}$ a Lévy process if it has independent and stationary increments, $X(0)=0$ a.s., it is stochastically continuous at any $t$, and almost all sample paths are right continuous and have left limits. In this paper, we need the Lévy process with time parameter running on $\mathbf{R}$. Let $\left\{X_{-}(t), t \geq 0\right\}$ be an independent copy of $\{X(t), t \geq 0\}$ and extend $\{X(t)\}$ in such a way that $X(t)=-X_{-}(-t+0)$ for $t<0$. If, furthermore, the distribution of $X(1)$ is symmetric $\alpha$-stable, $0<\alpha \leq 2$, in the sense that $E[\exp \{i \theta X(1)\}]=\exp \left\{-c|\theta|^{\alpha}\right\}, \theta \in \mathbf{R}$, for some $c>0$, we call $\{X(t)\}$ a symmetric $\alpha$-stable Lévy process, and denote it by $\left\{Z_{\alpha}(t), t \in\right.$ $\mathbf{R}$ ) throughout this paper. For simplicity, we always assume $c=1$. When $\alpha=2$, it is nothing but the standard Brownian motion up to a multiplicative constant.

For $0<H<1$ and $0<\alpha \leq 2$ such that $H \neq \frac{1}{\alpha}$, define a linear fractional stable motion $\left\{\Delta_{H, \alpha}(t), t \in \mathbf{R}\right\}$ by

$$
\Delta_{H, \alpha}(t)=\int_{-\infty}^{\infty}\left\{(t-s)_{+}^{H-1 / \alpha}-(-s)_{+}^{H-1 / \alpha}\right\} d Z_{\alpha}(s),
$$

where $x_{+}=\max (x, 0)$ and $0^{s}=0$ even for $s \leq 0$. When $\alpha=2$, it is the fractional Brownian motion (fBm). In this paper, we are concerned with the case $\alpha<2$. Thus we always assume

Received August 17, 2006; revised March 20, 2008

Key words: linear fractional stable motion, integral with respect to linear fractional stable motion, slowly varying function, weighted sum 
that $H>1 / \alpha$, because if $H<1 / \alpha$, almost all sample paths of $\Delta_{H, \alpha}(t)$ are nowhere bounded ([5]), which is not an interesting case. Note that $H>1 / \alpha$ implies $\alpha>1$, since $H<1$.

Let $\left\{X_{j}\right\}_{j \in \mathbf{Z}}$ be independent and identically distributed random variables such that

$$
\frac{1}{n^{1 / \alpha}} \sum_{j=1}^{n} X_{j} \stackrel{d}{\longrightarrow} Z_{\alpha}(1) \quad \text { as } n \rightarrow \infty,
$$

where $\stackrel{d}{\longrightarrow}$ denotes convergence in distribution. Since $\alpha>1,\left\{X_{j}\right\}$ have the finite first moment, and we assume $E\left[X_{j}\right]=0$. Define a sequence of strongly dependent random variables $\left\{\xi_{j}\right\}$ by

$$
\xi_{j}=\sum_{k \in \mathbf{Z}} c_{k} X_{j-k}, \quad j \in \mathbf{Z},
$$

where $\left\{c_{j}\right\}$ are suitably chosen depending on the problem. We want to find the class of $f$ 's and conditions for the convergence of

$$
I_{n}(f):=A_{n} \sum_{j \in \mathbf{Z}} f\left(\frac{j}{n}\right) \xi_{j}
$$

with a suitable scaling $A_{n}$ to

$$
I(f):=\int_{-\infty}^{\infty} f(u) d \Delta_{H, \alpha}(u)
$$

in distribution as $n \rightarrow \infty$.

This problem was studied by Pipiras and Taqqu [6] for the case $\alpha=2$. They gave sufficient conditions for the convergence

$$
\frac{1}{n^{H}} \sum_{j \in \mathbf{Z}} f\left(\frac{j}{n}\right) \xi_{j} \rightarrow \int_{\mathbf{R}} f(u) d B_{H}(u)
$$

in distribution as $n \rightarrow \infty$, where $B_{H}$ is a $\mathrm{fBm}$ with index $H \in(1 / 2,1)$. They also considered the following problem in [6]. If $\left\{f_{t}, t \geq 0\right\}$ are the kernel functions from the "moving average" representation of fBm with another index $H^{\prime}$, then the limit $\left\{I\left(f_{t}\right)\right\}$ in the sense of convergence of finite-dimensional distributions is a fBm with index $H+H^{\prime}-1 / 2$.

The purpose of this paper is to study the limit theorems of Pipiras and Taqqu [6] in the case $\alpha<2$. For it, we take linear fractional stable motions $\left\{\Delta_{H, \alpha}(t)\right\}$ instead of $\left\{B_{H}(t)\right\}$.

The rest of the paper is organized as follows. In Section 2, we propose a class of deterministic functions $f$ for which stochastic integrals with respect to linear fractional stable motions are well-defined (also see [3]). In Section 3, we present our first theorem of type (1.2) and the proof is given. In Section 4, we also show that if $\left\{f_{t}, t \geq 0\right\}$ are the kernel functions from the "moving average" representation of a linear fractional stable motion with another index $H^{\prime}$, then $\left\{I_{n}\left(f_{t}\right), t \geq 0\right\}$ converges to a linear fractional stable motion $C \Delta_{H+H^{\prime}-1 / \alpha, \alpha}(t)$ with some $C>0$, in the sense of finite-dimensional distributions. 


\section{Stochastic integrals}

Throughout the paper we denote by $f * g$ the usual convolution:

$$
(f * g)(s)=\int_{-\infty}^{\infty} f(s-u) g(u) d u
$$

for measurable functions $f$ and $g$.

In this section we define stochastic integrals with respect to $d \Delta_{H, \alpha}$. The following fact has been pointed out already in [3] by one of the authors of the present paper with other coauthors. Since the paper [3] was not published, we explain it here with the permission of two coauthors.

In the following, we put $\beta=H-1 / \alpha$ for notational convenience, and thus $\beta$ satisfies $0<\beta<1-1 / \alpha$. Then $\Delta_{H, \alpha}(t)$ is written as

$$
\Delta_{H, \alpha}(t)=\int_{-\infty}^{\infty}\left\{(t-s)_{+}^{\beta}-(-s)_{+}^{\beta}\right\} d Z_{\alpha}(s) .
$$

Define, for $\gamma>0$,

$$
\rho_{\gamma}(x):= \begin{cases}0, & x \geq 0 \\ \gamma|x|^{\gamma-1}, & x<0\end{cases}
$$

Then, since

$$
(t-s)_{+}^{\beta}-(-s)_{+}^{\beta}=\beta \int_{s}^{\infty} 1_{[0, t)}(u)(u-s)^{\beta-1} d u=\left(1_{[0, t)} * \rho_{\beta}\right)(s)
$$

(see [6]), (2.1) can be rewritten as

$$
\Delta_{H, \alpha}(t)=\int_{-\infty}^{\infty}\left(1_{[0, t)} * \rho_{\beta}\right)(s) d Z_{\alpha}(s)
$$

On the other hand, for the simple function of the form

$$
f(u)=\sum_{k=1}^{n} f_{k} 1_{\left[u_{k}, u_{k+1}\right)}(u), \quad f_{k} \in \mathbf{R}, \quad u_{k}<u_{k+1}, \quad k=1, \ldots, n,
$$

we should define

$$
\int_{-\infty}^{\infty} f(u) d \Delta_{H, \alpha}(u)=\sum_{k=1}^{n} f_{k}\left(\Delta_{H, \alpha}\left(u_{k+1}\right)-\Delta_{H, \alpha}\left(u_{k}\right)\right) .
$$

However, by (2.2), the right-hand side can be rewritten as

$$
\int_{-\infty}^{\infty}\left(f * \rho_{\beta}\right)(s) d Z_{\alpha}(s) .
$$

Thus we arrive at the following definition of $I(f)$. 
DEFINITION 2.1. Let

$$
\Lambda_{\alpha, \beta}=\left\{f:|f| * \rho_{\beta} \in L^{\alpha}\right\} .
$$

For $f \in \Lambda_{\alpha, \beta}$, we define

$$
I(f)=\int_{-\infty}^{\infty} f(u) d \Delta_{H, \alpha}(u):=\int_{-\infty}^{\infty}\left(f * \rho_{\beta}\right)(s) d Z_{\alpha}(s) .
$$

Clearly, $I(f)$ is linear with respect to $f$. Here, notice that the right-hand side of (2.3) is well-defined thanks to the assumption that $|f| * \rho_{\beta} \in L^{\alpha}$, and it holds

$$
E\left[e^{i \theta I(f)}\right]=\exp \left\{|\theta|^{\alpha}\left\|f * \rho_{\beta}\right\|_{L^{\alpha}}^{\alpha}\right\}, \quad \theta \in \mathbf{R} .
$$

We next study the class $\Lambda_{\alpha, \beta}$. By the Hausdorff-Young inequality, we immediately have

Lemma 2.2. Let $p \geq 1$.

(i) If $f \in L^{1} \cap L^{p}, g \in L^{1}$ and $h \in L^{p}$, then

$$
\|f *(g+h)\|_{L^{p}} \leq\|f\|_{L^{p}}\|g\|_{L^{1}}+\|f\|_{L^{1}}\|h\|_{L^{p}} .
$$

(ii) If $f_{n} \rightarrow f$ in $L^{1} \cap L^{p}, g_{n} \rightarrow g$ in $L^{1}$ and $h_{n} \rightarrow h$ in $L^{p}$, then

$$
f_{n} *\left(g_{n}+h_{n}\right) \rightarrow f *(g+h) \text { in } L^{p} .
$$

Now let $\alpha$ and $\beta$ be as before. Recall that $1<\alpha<2$ and $0<\beta<1-\frac{1}{\alpha}$.

THEOREM 2.3. Let $p>\frac{1}{1-\beta}$. (Note that the case $p=\alpha$ is included.) Then, there exists $C=C(\beta, p)<\infty$ such that for every $f \in L^{p} \cap L^{1}$,

$$
\left\|f * \rho_{\beta}\right\|_{L^{p}} \leq\|f\|_{L^{p}}+C\|f\|_{L^{1}},
$$

and, therefore, (taking $p=\alpha$ ), we have

$$
L^{p} \cap L^{1} \subset \Lambda_{\alpha, \beta} .
$$

ProOF. Let $g(x)=\rho_{\beta}(x) 1_{(-1,0)}(x)$ and $h(x)=\rho_{\beta}(x) 1_{(-\infty,-1]}(x)$. Then $\beta-1>-1$ and $p(\beta-1)<-1$ imply $g \in L^{1}$ and $h \in L^{p}$, respectively. In fact, $\|g\|_{L^{1}}=1$. Therefore, applying the previous Lemma, we have

$$
\left\|f * \rho_{\beta}\right\|_{L^{p}} \leq\|f\|_{L^{p}}+\|f\|_{L^{1}}\|h\|_{L^{p}},
$$

which is (2.4).

The following corollaries follow easily from Theorem 2.3.

COROLlaRY 2.4. Let $f(x), x \in \mathbf{R}$, be a measurable function vanishing outside a compact set. If there exist constants $C>0, a \in \mathbf{R},-\frac{1}{\alpha}<\gamma<0$ such that

$$
|f(x)| \leq C|x-a|^{\gamma}, \quad x \in \mathbf{R},
$$

then $f \in \Lambda_{\alpha, \beta}$. 
COROLlary 2.5. If $f(x), x \in \mathbf{R}$, is a bounded, integrable function, then $f \in \Lambda_{\alpha, \beta}$.

Now we discuss another important function belonging to $\Lambda_{\alpha, \beta}$. This function will be treated again in Section 4. We also mention the fact (Theorem 2.6), which is not used in this section but will be used in Section 4.

For $\gamma \in \mathbf{R}$ and $t \in \mathbf{R}$, we put

$$
f_{t, \gamma}(u)= \begin{cases}(t-u)_{+}^{\gamma}-(-u)_{+}^{\gamma}, & \gamma \neq 0, \\ 1_{(0, t]}(u), & \gamma=0, \quad u \in \mathbf{R} .\end{cases}
$$

Recall that this function appeared in (2.1):

$$
\Delta_{H, \alpha}(t)=\int_{-\infty}^{\infty} f_{t, \beta}(u) d Z_{\alpha}(u), \quad \beta=H-1 / \alpha(>0) .
$$

If $-\frac{1}{\alpha}<\gamma<0$, then applying Corollaries 2.4 and 2.5 to $f_{t, \gamma} 1_{[-A, A]}$ and $f_{t, \gamma} 1_{[-A, A]^{c}}$, respectively, we see that $f_{t, \gamma} \in \Lambda_{\alpha, \beta}$, and hence its stochastic integral is defined and can be calculated explicitly as follows.

THEOREM 2.6. If $-\frac{1}{\alpha}<\gamma<0$, then $f_{t, \gamma} \in \Lambda_{\alpha, \beta}$ and it holds that

$$
\int_{-\infty}^{\infty} f_{t, \gamma}(u) d \Delta_{H, \alpha}(u)=\beta B(\gamma+1, \beta) \Delta_{H+\gamma, \alpha}(t),
$$

where $B(\cdot, \cdot)$ is the usual beta function.

PROOF. By a direct calculation we see that

$$
f_{t, \gamma} * \rho_{\beta}=\beta B(\gamma+1, \beta) f_{t, \beta+\gamma}
$$

provided that $\beta-1<\gamma<0$. Therefore, it is easy to see that $f_{t, \gamma} * \rho_{\beta} \in L^{\alpha}$. Also, by the definition of the stochastic integral, we can deduce that

$$
\begin{aligned}
\int_{-\infty}^{\infty} f_{t, \gamma}(u) d \Delta_{H, \alpha}(u) & =\int_{-\infty}^{\infty}\left(f_{t, \gamma} * \rho_{\beta}\right)(u) d Z_{\alpha}(u) \\
& =\beta B(\gamma+1, \beta) \int_{-\infty}^{\infty} f_{t, \beta+\gamma}(u) d Z_{\alpha}(u)
\end{aligned}
$$

The first equality is the definition and the second follows from (2.6). However, the extreme right-hand side equals $\beta B(\gamma+1, \beta) \Delta_{H+\gamma, \alpha}(t)$ by (2.5).

\section{Convergence to stochastic integrals}

Our first result in the present paper is the following. 
THEOREM 3.1. Let $1<\alpha<2$ and $1 / \alpha<H<1$. Recall that we put $\beta=H-\frac{1}{\alpha}$, let $c_{j}=\beta j^{\beta-1} L(j), j=1,2,3, \ldots$, for some slowly varying function $L(x), x>0$, and define

$$
\xi_{j}=\sum_{k=1}^{\infty} c_{k} X_{j-k}, \quad j \in \mathbf{Z}
$$

where $\left\{X_{j}\right\}_{j=-\infty}^{\infty}$ is a sequence of independent identically distributed random variables introduced in (1.1).Then

$$
\frac{1}{n^{H} L(n)} \sum_{j \in \mathbf{Z}} f\left(\frac{j}{n}\right) \xi_{j} \stackrel{d}{\longrightarrow} \int_{-\infty}^{\infty} f(u) d \Delta_{H, \alpha}(u) \quad \text { as } n \rightarrow \infty
$$

for every piece-wise continuous function $f(x), x \in \mathbf{R}$, such that

$$
|f(u)| \ll(1+|u|)^{-(1+\varepsilon)} \text { for some } \varepsilon>0 .
$$

Here $|f(u)| \ll g(u)$ means that there exists $K>0$ satisfying $|f(u)| \leq K g(u)$ for all $u \in \mathbf{R}$.

REMARK 3.2. Note that $\left\{c_{j}\right\}$ above satisfies that $\sum_{j=1}^{\infty}\left|c_{j}\right|^{a}<\infty$ for some $a<\alpha$. Then the sequence of random variables $\left\{\xi_{j}\right\}_{j \in \mathbf{Z}}$ belongs to the domain of attraction of $\Delta_{H, \alpha}(t)$, because it satisfies the conditions (a) and (b) in the following result, which is a known result on the domain of attraction of the linear fractional stable process $\Delta_{H, \alpha}(t)$. See the theorem below. Therefore, if $f$ is a simple function with compact support, our assertion is already known, although we do not use it here, and our purpose here is to extend it for more general functions.

THEOREM 3.3 ([4]). Let $1<\alpha \leq 2,1 / \alpha<H<1$ and let $\left\{c_{j}\right\}_{j=-\infty}^{\infty}$ be a sequence of real numbers such that $\sum_{j}\left|c_{j}\right|^{a}<\infty$ for some $a<\alpha$. Suppose that there exists an $L(n)$ which is slowly varying at $\infty$ such that

(a) $\lim _{n \rightarrow \infty} \frac{1}{n^{\beta} L(n)} \sum_{j=1}^{n} c_{j}=1$,

(b) $\quad c_{j}=O\left(j^{\beta-1} L(j)\right)$ as $j \rightarrow \infty$.

Then

$$
\frac{1}{n^{H} L(n)} \sum_{j=1}^{[n t]} \xi_{j} \stackrel{f . d .}{\longrightarrow} \Delta_{H, \alpha}(t) \quad \text { as } n \rightarrow \infty
$$

where $\stackrel{f . d .}{\longrightarrow}$ means convergence of all finite dimensional distributions.

We next proceed to the proof of Theorem 3.1. First, notice that

$$
\sum_{j \in \mathbf{Z}} f\left(\frac{j}{n}\right) \xi_{j}=\sum_{j \in \mathbf{Z}} f\left(\frac{j}{n}\right) \sum_{k=1}^{\infty} c_{k} X_{j-k}=\sum_{m \in \mathbf{Z}} \sum_{j=m+1}^{\infty} f\left(\frac{j}{n}\right) c_{j-m} X_{m}
$$


Therefore, putting

$$
g_{n}(s):=\sum_{j>[n s]} f\left(\frac{j}{n}\right) \frac{c_{j-[n s]}}{n^{\beta} L(n)},
$$

we can rewrite the sum as follows:

$$
\frac{1}{n^{H} L(n)} \sum_{j \in \mathbf{Z}} f\left(\frac{j}{n}\right) \xi_{j}=\frac{1}{n^{1 / \alpha}} \sum_{j \in \mathbf{Z}} g_{n}\left(\frac{j}{n}\right) X_{j} .
$$

Thus the weighted sum of $\xi_{j}$ is represented by that of $X_{j}$. The convergence of the latter is already discussed and our main tool here is the following theorem, which is essentially due to [4] although the conditions are modified a little. A similar result is also found in Avram and Taqqu [1].

THEOREM 3.4 ([4]). Let $\left\{X_{j}\right\}$ be random variables in (1.1) and suppose that functions $g, g_{1}, g_{2}, \ldots$ defined on $\mathbf{R}$ satisfy the following conditions:

(A) $g_{n} \stackrel{\text { c.c. }}{\rightarrow} g \quad$ a.e. as $n \rightarrow \infty$, where $g_{n}(u) \stackrel{\text { c.c. }}{\rightarrow} g(u)$ at $u=u_{0}$ means that $g_{n}\left(u_{n}\right) \rightarrow$ $g\left(u_{0}\right)$ whenever $u_{n} \rightarrow u_{0}$.

(B) $\tilde{g}_{n} \longrightarrow g$ as $n \rightarrow \infty$ in $L^{\alpha+\varepsilon} \cap L^{\alpha-\varepsilon}$ for some $\varepsilon>0$, where $\tilde{g}_{n}(u)=$ $g_{n}([n u] / n), u \in \mathbf{R}, n=1,2,3, \ldots$.

Then we have

$$
\frac{1}{n^{1 / \alpha}} \sum_{j \in \mathbf{Z}} g_{n}\left(\frac{j}{n}\right) X_{j} \stackrel{d}{\longrightarrow} \int_{-\infty}^{\infty} g(u) d Z_{\alpha}(u) \text { as } n \rightarrow \infty .
$$

We shall see that $\left\{g_{n}\right\}_{n}$ in (3.1) satisfy the conditions (A) and (B) with $g=f * \rho_{\beta}$. Let $\phi(x)=\beta x^{\beta-1} L(x)(x \geq 0)$ so that $c_{j}=\phi(j)(j \in \mathbf{N})$. Define

$$
\rho^{(n)}(x)= \begin{cases}\frac{c_{[-n x]}}{n^{\beta-1} L(n)}, & x<0, \\ 0, & x \geq 0 .\end{cases}
$$

Then we have for $x \neq 0$,

$$
\rho^{(n)}(x) \stackrel{\text { c.c. }}{\longrightarrow} \rho_{\beta}(x) \text { as } n \rightarrow \infty,
$$

where $\rho_{\beta}(x)$ is the same as before. Since $\phi$ is regularly varying, for any $\varepsilon>0$, there exists a $C>0$ such that

$$
\frac{\phi(x)}{\phi(y)} \leq C\left(\frac{x}{y}\right)^{\beta-1}\left\{\left(\frac{x}{y}\right)^{\varepsilon}+\left(\frac{x}{y}\right)^{-\varepsilon}\right\}
$$

for all sufficiently large $x, y>0$. Since it is harmless to change the values of finitely many $c_{j}$ 's, we may, without loss of generality, modify the value of $\phi(x)$ in a neighborhoods of the origin. Therefore, we may assume that

$$
\rho^{(n)}(x) \leq C\left(\rho_{\beta+\varepsilon}(x)+\rho_{\beta-\varepsilon}(x)\right)
$$


holds for all $x \in \mathbf{R}$ and all sufficiently large $n$.

Now we prepare the following two lemmas to check that the above $g_{n}$ satisfies the conditions (A) and (B) in Theorem 3.4.

Lemma 3.5. Let $p>\frac{1}{1-\beta}$. (Note that the case $p=\alpha$ is included.) If $\varphi_{n} \rightarrow \varphi$ in $L^{p} \cap L^{1}$, then

$$
\varphi_{n} * \rho^{(n)} \rightarrow \varphi * \rho_{\beta} \quad \text { in } L^{p} .
$$

Proof. Let $\rho_{1}^{(n)}=\rho^{(n)} 1_{(-1,0)}$. Then $\rho_{1}^{(n)} \rightarrow \rho_{\beta} 1_{(-1,0)}$, where the convergence is in $L^{1}$ because $\rho_{1}^{(n)}(x) \leq C|x|^{\beta-1-\varepsilon}$ and $\beta>0$. Similarly, let $\rho_{2}^{(n)}=\rho^{(n)} 1_{(-\infty,-1]}$. Then $\rho_{2}^{(n)} \rightarrow \rho_{\beta} 1_{(-\infty,-1]}$, where the convergence is in $L^{p}$ because $\rho_{2}^{(n)}(x) \leq C|x|^{\beta-1+\varepsilon}$ and $p(\beta-1+\varepsilon)<-1$. Therefore (3.2) follows from Lemma 2.2.

LEMMA 3.6. Let $\varphi_{n}$ and $\varphi$ be measurable functions satisfying

(i) $\varphi_{n} \stackrel{\text { c.c. }}{\longrightarrow} \varphi$ at all continuity points of $\varphi$, and

(ii) there exits a constant $C>0$ and an $F \in L^{1}$ such that

$$
\left|\varphi_{n}(x)\right| \leq C \text { and }\left|\varphi_{n}(x)\right| \leq F(x) .
$$

Then

$$
\varphi_{n} * \rho^{(n)} \stackrel{\text { c.c. }}{\longrightarrow} \varphi * \rho_{\beta} \quad \text { a.e. }
$$

Proof. Let $\rho_{1}^{(n)}$ and $\rho_{2}^{(n)}$ be as in the proof of the previous lemma. If $x_{n} \rightarrow x$, then $\varphi_{n}\left(x_{n}-u\right) \rho^{(n)}(u) \rightarrow \varphi(x-u) \rho_{\beta}(u)$ a.e. Therefore,

$$
\left(\varphi_{n} * \rho_{1}^{(n)}\right)\left(x_{n}\right)=\int_{-1}^{0} \varphi_{n}\left(x_{n}-u\right) \rho^{(n)}(u) d u \rightarrow \int_{-1}^{0} \varphi(x-u) \rho_{\beta}(u) d u
$$

because

$$
\left|\varphi_{n}\left(x_{n}-u\right) \rho^{(n)}(u)\right| \leq C|u|^{\beta-1-\varepsilon}, \quad-1<u<0 .
$$

(Choose $\varepsilon>0$ so that $\beta-\varepsilon>0$.) Similarly,

$$
\left(\varphi_{n} * \rho_{2}^{(n)}\right)\left(x_{n}\right)=\int_{-\infty}^{\infty} \varphi_{n}(u) \rho_{2}^{(n)}\left(x_{n}-u\right) d u \rightarrow \int_{-\infty}^{\infty} \varphi(u)\left(\rho_{\beta} 1_{(-\infty,-1]}\right)(x-u) d u,
$$

since

$$
\left|\varphi_{n}(u) \rho_{2}^{(n)}\left(x_{n}-u\right)\right| \leq \text { const. } F(u) .
$$

(Notice that $\rho_{2}^{(n)}$ is bounded). Thus we have

$$
\left(\varphi_{n} * \rho^{(n)}\right)\left(x_{n}\right)=\left(\varphi_{n} * \rho_{1}^{(n)}\right)\left(x_{n}\right)+\left(\varphi_{n} * \rho_{2}^{(n)}\right)\left(x_{n}\right) \rightarrow\left(\varphi * \rho_{\beta}\right)(x),
$$


which is (3.3).

Now we are ready to prove Theorem 3.1. Since for $s \in \mathbf{R}$,

$$
\tilde{g}_{n}(s)=g_{n}(s)=\sum_{j=1}^{\infty} f\left(\frac{j+[n s]}{n}\right) \frac{c_{j}}{n^{\beta} L(n)}=\int_{-\infty}^{\infty} f\left(\frac{[n s]}{n}-\frac{[n x]}{n}\right) \rho^{(n)}(x) d x,
$$

we can rewrite as

$$
g_{n}(s)=\left(f_{n} * \rho^{(n)}\right)([n s] / n) \quad \text { where } \quad f_{n}(x)=f([n x] / n) .
$$

It follows that $f$ in Theorem 3.1 and the above $f_{n}$ satisfy the conditions of $\varphi_{n}$ and $\varphi$ in Lemmas 3.5 and 3.6. Furthermore, $\varphi_{n} * \rho^{(n)}$ and $\varphi * \rho_{\beta}$ in the previous two lemmas can be replaced with $g_{n}=f_{n} * \rho^{(n)}$ and $g=f * \rho_{\beta}$ in Theorem 3.4, respectively. Therefore, by noting the following proposition derived easily from Lemmas 3.5 and 3.6, the proof of Theorem 3.1 is completed by Theorem 3.4.

PROPOSITION 3.7. Let $g_{n}$ be the functions defined by (3.1). Then, under the conditions of Theorem 3.1,

$$
g_{n} \stackrel{c . c .}{\longrightarrow} f * \rho_{\beta} \quad \text { a.e. }
$$

Furthermore, the convergence also holds in $L^{\alpha+\varepsilon} \cap L^{\alpha-\varepsilon}$ for some $\varepsilon>0$.

\section{The case that the limit is another linear fractional stable motion}

In this section, we extend the following known story for $\mathrm{fBm}$ ([7]) to the case of the linear fractional stable motion. It is shown in [7] that if $\left\{f_{t}, t \geq 0\right\}$ are the kernel functions from the "moving average" representation of a $\mathrm{fBm}$ with index $H^{\prime}$, then the limit is a $\mathrm{fBm}$ with index $H+H^{\prime}-\frac{1}{2}$. We can now ask what happens if we take $\left\{\Delta_{H, \alpha}(t)\right\}$ instead of $\left\{B_{H}(t)\right\}$. The following theorem gives us an answer.

THEOREM 4.1. Let $1<\alpha<2,1 / \alpha<H<1,0<H^{\prime}<1 / \alpha, \beta^{\prime}=H^{\prime}-1 / \alpha$ and

$$
f_{t, \beta^{\prime}}(u)=(t-u)_{+}^{\beta^{\prime}}-(-u)_{+}^{\beta^{\prime}}, u \in \mathbf{R} .
$$

Suppose that the sequence $\left\{\xi_{j}\right\}_{j \in \mathbf{Z}}$ satisfies the conditions of Theorem 3.1. Then, the processes

$$
\frac{1}{n^{H} L(n)} \sum_{j \in \mathbf{Z}} f_{t, \beta^{\prime}}\left(\frac{j}{n}\right) \xi_{j}, \quad t \in \mathbf{R}
$$

converge (up to constant) to the linear fractional stable motion $\left\{\beta B\left(\beta^{\prime}+1, \beta\right)\right.$ $\left.\Delta_{H+H^{\prime}-1 / \alpha, \alpha}(t), t \in \mathbf{R}\right\}$ in the sense of finite-dimensional distributions.

Proof. Put

$$
Y_{n}(t)=\frac{1}{n^{H} L(n)} \sum_{j \in \mathbf{Z}} f_{t, \beta^{\prime}}\left(\frac{j}{n}\right) \xi_{j}, \quad t \in \mathbf{R}
$$


and

$$
Y(t)=\int_{-\infty}^{\infty} f_{t, \beta^{\prime}}(s) d \Delta_{H, \alpha}(s), \quad t \in \mathbf{R} .
$$

Since $-\frac{1}{\alpha}<\beta^{\prime}<0$, it follows from Theorem 2.6 that $f_{t, \beta^{\prime}} \in \Lambda_{\alpha, \beta}$ and

$$
\int_{-\infty}^{\infty} f_{t, \beta^{\prime}}(s) d \Delta_{H, \alpha}(s)=\int_{-\infty}^{\infty}\left(f_{t, \beta^{\prime}} * \rho_{\beta}\right)(s) d Z_{\alpha}(s)=\beta B\left(\beta^{\prime}+1, \beta\right) \Delta_{H+H^{\prime}-1 / \alpha, \alpha}(t) .
$$

We are going to show that as $n \rightarrow \infty, Y_{n}(t)$ converges to $Y(t)$ in the sense of finitedimensional distributions. By the Cramer-Wold method (a random vector converges in distribution if and only if all linear combinations of its elements do), it is enough to show that for each $t \in \mathbf{R}, Y_{n}(t)$ converges to $Y(t)$ in distribution.

To prove it, notice that we can rewrite the sum as follows:

$$
\frac{1}{n^{H} L(n)} \sum_{j \in \mathbf{Z}} f_{t, \beta^{\prime}}\left(\frac{j}{n}\right) \xi_{j}=\frac{1}{n^{1 / \alpha}} \sum_{j \in \mathbf{Z}} g_{n, t}\left(\frac{j}{n}\right) X_{j},
$$

where

$$
g_{n, t}(s):=\sum_{j>[n s]} f_{t, \beta^{\prime}}\left(\frac{j}{n}\right) \frac{c_{j-[n s]}}{n^{\beta} L(n)} .
$$

Moreover we can rewrite as

$$
\begin{aligned}
g_{n, t}(s) & =\sum_{j=1}^{\infty} f_{t, \beta^{\prime}}\left(\frac{j+[n s]}{n}\right) \frac{c_{j}}{n^{\beta} L(n)}=\int_{-\infty}^{\infty} f_{t, \beta^{\prime}}\left(\frac{[n s]}{n}-\frac{[n x]}{n}\right) \rho^{(n)}(x) d x \\
& =\left(f_{t, \beta^{\prime}}^{(n)} * \rho^{(n)}\right)([n s] / n),
\end{aligned}
$$

where $f_{t, \beta^{\prime}}^{(n)}(x)=f_{t, \beta^{\prime}}([n x] / n)$. We shall show that $g_{n, t}=f_{t, \beta^{\prime}}^{(n)} * \rho^{(n)}$ satisfies the conditions (A) and (B) in Theorem 3.4 with $g=f_{t, \beta^{\prime}} * \rho_{\beta}$ in a similar way as before. However, this time, we cannot apply Lemma 3.6, because $f_{t, \beta^{\prime}}(u)$ is not bounded. Thus we need the following lemma, which is a modification of Lemma 3.6.

Lemma 4.2. Suppose $\varphi_{n}$ and $\varphi$ are measurable functions such that

(i) $\varphi_{n} \stackrel{\text { c.c. }}{\longrightarrow} \varphi$ at all continuity points of $\varphi$, and

(ii) there exists a constant $C>0$ and $\gamma(-1<\gamma<-1 / \alpha)$ satisfying

$$
\left|\varphi_{n}(x)\right| \leq \begin{cases}C|x|^{\gamma}, & -|t|<x<0 \\ 0, & \text { otherwise }\end{cases}
$$

Then

$$
\varphi_{n} * \rho^{(n)} \stackrel{\text { c.c. }}{\longrightarrow} \varphi * \rho_{\beta} \quad \text { a.e. }
$$


Proof. Let $\rho_{1}^{(n)}, \rho_{2}^{(n)}$ be as in the proof of Lemma 3.5. By (i), if $x_{n} \rightarrow x$, then $\varphi_{n}\left(x_{n}-u\right) \rho^{(n)}(u) \rightarrow \varphi(x-u) \rho_{\beta}(u)$, a.e. Therefore

$$
\left(\varphi_{n} * \rho_{2}^{(n)}\right)\left(x_{n}\right)=\int_{-\infty}^{\infty} \varphi_{n}(u) \rho_{2}^{(n)}\left(x_{n}-u\right) d u \rightarrow \int_{-\infty}^{\infty} \varphi(u) \rho_{\beta} 1_{(-\infty,-1]}(x-u) d u
$$

because

$$
\left|\varphi_{n}(u) \rho_{2}^{(n)}\left(x_{n}-u\right)\right| \leq \text { const. }|u|^{\gamma} \quad(\gamma>-1) .
$$

(Notice that $\rho_{2}^{(n)}$ is bounded.) Next we shall show

$$
\left(\varphi_{n} * \rho_{1}^{(n)}\right)\left(x_{n}\right)=\int_{-1}^{0} \varphi_{n}\left(x_{n}-u\right) \rho^{(n)}(u) d u \rightarrow \int_{-1}^{0} \varphi(x-u) \rho_{\beta}(u) d u
$$

by dividing the integral into three cases: (1) $0<x$, (2) $-1>x$ and (3) $-1<x<0$.

Case (1) is easily verified, because $\varphi_{n}\left(x_{n}-u\right) \rho^{(n)}(u)=0$. Case (2) is the same case as Lemma 3.6, since $\varphi_{n}$ is bounded on $-1<u<0$ and it also belongs to $L^{1}$. As to Case (3), let $u=y_{n}$ be an intersection point of $\left|x_{n}-u\right|^{\gamma}$ and $\rho_{1}^{(n)}(u)$, and divide the integral into two parts as follows:

$$
\int_{-1}^{0} \varphi_{n}\left(x_{n}-u\right) \rho^{(n)}(u) d u=\left(\int_{x_{n}}^{y_{n}}+\int_{y_{n}}^{0}\right) \varphi_{n}\left(x_{n}-u\right) \rho^{(n)}(u) d u=I_{1}+I_{2} .
$$

When $x_{n} \leq u \leq y_{n}$, it follows that

$$
\left|\varphi_{n}\left(x_{n}-u\right) \rho^{(n)}(u)\right| \leq \text { const. }\left|x_{n}-u\right|^{\gamma} \quad(\gamma>-1)
$$

since $\rho_{1}^{(n)}$ is bounded. When $y_{n} \leq u \leq 0$, it follows that

$$
\left|\varphi_{n}\left(x_{n}-u\right) \rho^{(n)}(u)\right| \leq \text { const. }|u|^{\beta-1-\varepsilon}
$$

(Note that $\beta-1-\varepsilon>-1$ ). Therefore Case (3) is verified. Thus we have

$$
\left(\varphi_{n} * \rho^{(n)}\right)\left(x_{n}\right)=\left(\varphi_{n} * \rho_{1}^{(n)}\right)\left(x_{n}\right)+\left(\varphi_{n} * \rho_{2}^{(n)}\right)\left(x_{n}\right) \rightarrow\left(\varphi * \rho_{\beta}\right)(x) .
$$

The following lemma plays the same role as Proposition 3.7 in the proof of Theorem 4.1. Therefore, once we could prove Lemma 4.3, we can complete the proof of Theorem 4.1.

LEMmA 4.3. Let $g_{n, t}$ be the functions defined by (4.2). Then, under the conditions of Theorem 4.1,

$$
g_{n, t} \stackrel{c . c .}{\longrightarrow} f_{t, \beta^{\prime}} * \rho_{\beta} \quad \text { a.e. }
$$

Furthermore, the convergence also holds in $L^{\alpha+\varepsilon} \cap L^{\alpha-\varepsilon}$ for some $\varepsilon>0$. 
Proof. We check that $g_{n, t}=f_{t, \beta^{\prime}}^{(n)} * \rho^{(n)} \rightarrow g=f_{t, \beta^{\prime}} * \rho_{\beta}$ in $L^{\alpha+\varepsilon} \cap L^{\alpha-\varepsilon}$ and in the sense of ' $\stackrel{\text { c.c. }}{\longrightarrow}$. To prove $f_{t, \beta^{\prime}}^{(n)} * \rho^{(n)} \rightarrow f_{t, \beta^{\prime}} * \rho_{\beta}$ in $L^{\alpha+\varepsilon} \cap L^{\alpha-\varepsilon}$, it is enough to show that $f_{t, \beta^{\prime}}^{(n)} \rightarrow f_{t, \beta^{\prime}}$ in $L^{p} \cap L^{1}$ by Lemma 3.5. Since $\left|f_{t, \beta^{\prime}}^{(n)}(\cdot)\right|$ are increasing functions, it follows that

$$
\left|f_{t, \beta^{\prime}}^{(n)}(u)\right| \leq\left|f_{t, \beta^{\prime}}(u)\right| .
$$

Furthermore, since it follows that $f_{t, \beta^{\prime}} \in L^{p} \cap L^{1}$ and we can verify

$$
\lim _{n \rightarrow \infty} f_{t, \beta^{\prime}}^{(n)}(u)=f_{t, \beta^{\prime}}(u) \quad \text { a.e. }
$$

and we have that $f_{t, \beta^{\prime}}^{(n)} \rightarrow f_{t, \beta^{\prime}}$ in $L^{p} \cap L^{1}$, which implies that $f_{t, \beta^{\prime}}^{(n)} * \rho^{(n)} \rightarrow f_{t, \beta^{\prime}} * \rho_{\beta}$ in $L^{\alpha+\varepsilon} \cap L^{\alpha-\varepsilon}$, by Lemma 3.5 as mentioned.

Next, we prove the convergence in the sense of ' $\stackrel{\text { c.c. } .}{\longrightarrow}$. For this purpose, it is enough to check that $f_{t, \beta^{\prime}}^{(n)}$ and $f_{t, \beta^{\prime}}$ satisfy conditions (i) and (ii) in Lemma 3.6 or Lemma 4.2 with the replacement of $\varphi_{n}$ and $\varphi$ by $f_{t, \beta^{\prime}}^{(n)}$ and $f_{t, \beta^{\prime}}$, respectively. As to condition (i), it is trivial because $f_{t, \beta^{\prime}}(u)$ is piece-wise continuous. As to condition (ii), observe that $f_{t, \beta^{\prime}}$ explodes around $u=t$ and $u=0$. Suppose that $t<0$. (In the case that $t \geq 0$, the proof is similar, so that we omit the detail.) We can divide $f_{t, \beta^{\prime}}$ into four parts:

$$
\begin{gathered}
f_{t, \beta^{\prime}}(u) \cdot 1_{(-\infty, 2 t)}(u)+f_{t, \beta^{\prime}}(u) \cdot 1_{[2 t, t)}(u)+f_{t, \beta^{\prime}}(u) \cdot 1_{[t, 0)}(u)+f_{t, \beta^{\prime}}(u) \cdot 1_{[0, \infty)}(u) \\
=: f_{1}(u)+f_{2}(u)+f_{3}(u)+0 .
\end{gathered}
$$

It follows that $f_{2}(u)$ and $f_{3}(u)$ satisfy the condition (ii) in Lemma 4.2, since $\beta^{\prime}>\gamma$. As to $f_{1}(u)$, note that $f_{t, \beta^{\prime}}(u)=O\left(|u|^{\beta^{\prime}-1}\right)$ as $|u| \rightarrow \infty$. Therefore $f_{t, \beta^{\prime}}(u)$ is bounded and $\left|f_{t, \beta^{\prime}}(u)\right| \leq F(u)$, where $F \in L^{1}$, because $\beta^{\prime}<0$. Applying Lemma 3.6, we have that $g_{n, t} \stackrel{\text { c.c. }}{\longrightarrow} f_{t, \beta^{\prime}} * \rho_{\beta}$ a.e.

ACKNowledgement. The authors would like to thank Yuji Kasahara for his many valuable comments during this work. They are also grateful to an associate editor for his/her helpful comments on the final version of the paper.

\section{References}

[1] AVRAM, F. and TAQQU, M. S., Weak convergence of moving averages with infinite variance, in EBERLEIN, E. and TAQQU, M. S. (eds.), Dependence in Probability and Statistics, Birkauser, Boston, 399-415 (1986).

[2] Bingham, N. H., Goldie, C. M. and Teugels, J. L., Regular Variation, New York, Cambridge Univ. Press, 1987.

[ 3 ] Irisawa, M., Maejima, M. and Shimomura, S., A limit theorem for weighted sums of infinite variance random variables with long-range dependence, KSTS/RR-03/005, Dept. of Math., Keio Univ., 2003.

[4] Kasahara, Y. and Maejima, M., Weighted sums of i.i.d. random variables attracted to integrals of stable processes, Probab. Th. Rel. Fields 78, 75-96 (1988). 
[ 5 ] Maejima, M., A self-similar process with nowhere bounded sample paths, Z. Wahrscheinlichkeitstheorie verw. Gebiete 65, 115-119 (1983)

[ 6 ] PIPIRAS, V. and TAQQU, M. S., Convergence of weighted sums of random variables with long-range dependence, Stoch. Proc. Appl. 90, 157-174 (2000).

[ 7 ] Samorodnitsky, G. and Taqqu, M. S., Stable Non-Gaussian Processes, London, Chapman and Hall, 1994.

Present Addresses:

MAKOTO MAEJIMA

DEPARTMENT OF MATHEMATICS,

KEIO UNIVERSITY,

HIYOSHI, YOKOHAMA, 223-8522 JAPAN.

e-mail:maejima@math.keio.ac.jp

SAKURAKO SUZUKI

Graduate SCHOOL of SCIENCE AND Humanities,

OCHANOMIZU UNIVERSITY,

TOKYO, 112-8610 JAPAN.

e-mail: Suzukisakurako@aol.com 